\title{
COMPARISON OF PETROPHYSICAL PROPERTIES OF PREDOMINANT ROCKS FROM THE ARCHAEAN SECTION OF THE KOLA SUPERDEEP (SG-3)
}

\author{
Olga TRISHINA, Feliks GORBATSEVICH * and Mikhail KOVALEVSKIY
}

Geological Institute, KSC RAS, 14 Fersman St., Apatity 184209, Murmansk region, Russia

*Corresponding author's e-mail: gorich@geoksc.apatity.ru

\begin{tabular}{|c|c|}
\hline ARTICLE INFO & ABSTRACT \\
\hline Article history: & \multirow{11}{*}{$\begin{array}{l}\text { The values of density and velocity in the samples of the predominant rocks from the Archaean } \\
\text { section of the Kola superdeep borehole (SG-3) drilled in the northern frame of the Pechenga } \\
\text { palaeorift structure have been studied. The main part of rocks of the SG-3 Archaean section is } \\
\text { represented by gneisses, schists and amphibolites. Their main rock-forming minerals are } \\
\text { plagioclase, hornblende, mica and quartz. The texture of the rocks is mainly medium-grained, the } \\
\text { structure is nematogranoblastic and lepidogranoblastic. The parameters of elastic anisotropy } \\
\text { were estimated by the acoustopolariscopy method, the density and velocity of compression and } \\
\text { shear waves were determined in the lab and the mentioned characteristics were calculated by } \\
\text { mineral composition. The measured sample velocities are unusually low, which can be explained } \\
\text { by the decompaction effect of deep rocks. The values of the velocity characteristics that are close } \\
\text { to those for the conditions of the rock deep occurrence were obtained by calculation with regard } \\
\text { to their specific mineral composition. The average values of the velocity of compression and } \\
\text { shear waves for gneisses were calculated by mineral composition are } 6.38 \pm 0.16 \mathrm{~km} / \mathrm{s} \text { and } 3.52 \\
\pm 0.14 \mathrm{~km} / \mathrm{s} \text {, respectively. The average of the compression wave velocity for schists is } 6.40 \\
\pm 0.13 \mathrm{~km} / \mathrm{s} \text {, of the shear wave velocity }-3.46 \pm 0.09 \mathrm{~km} / \mathrm{s} \text { and for amphibolites } 6.84 \pm 0.13 \mathrm{~km} / \mathrm{s} \\
\text { and } 3.82 \pm 0.08 \mathrm{~km} / \mathrm{s} \text {, respectively. The dynamic moduli in different rocks are lower in schists, } \\
\text { medium in gneisses and higher in amphibolites. }\end{array}$} \\
\hline Received 4 & \\
\hline tember 2018 & \\
\hline Available online 25 September 2018 & \\
\hline Keywords: & \\
\hline Kola superdeep borehole & \\
\hline Archaean deep rocks & \\
\hline Properties & \\
\hline Densities & \\
\hline Compression & \\
\hline Shear wave velocities & \\
\hline
\end{tabular}

\section{BRIEF DESCRIPTION OF THE SECTION}

The Kola superdeep well (SG-3) is located in the northwest of the Kola Peninsula in the northern frame of the Pechenga structure (Fig. 1). It was drilled in the northern limb of the Pechenga geosyncline composed of rhythmically interbedded volcanogenic and tuffaceous-sedimentary strata extending to the NW at $300-310$ and dipping to the SW at angles of 30-50 (Kozlowsky, 1987). The SG-3 geological section whose pilot borehole reached $12262 \mathrm{~m}$ is represented by two complexes - Proterozoic and Archaean.

The following metamorphism facies and subfacies are distinguished in the borehole section:

1. Prehnite-pumpellyite facies (9-1400 m).

2. Greenschist facies (1400-4900 m):

a) epidote-chlorite subfacies (1400-3200 m);

b) biotite-actinolite subfacies (3200-4900 m).

3. Epidote-amphibolite facies (4900-6000 m).

4. Amphibolite facies (6000-12000 $\mathrm{m}$ and deeper).

Isotopic dating of the Pechenga rocks from SG-3 was performed by the potassium-argon and rubidiumstrontium methods. Biotite extracted from various depths yielded an age of 1600 to $3130 \mathrm{Ma}$, plagioclase -920 to $2100 \mathrm{Ma}$, microcline and amphibole 460$1960 \mathrm{Ma}$. According to the K-Ar and Rb-Sr data, progressive zonal metamorphism of Pechenga and retrograde metamorphism of occurring below Archaean rocks yielded an age of 1.7-2.1 Ma. (Hanski et al., 1990; Bayanova et al., 2002).

The Proterozoic complex $(0-6842 \mathrm{~m})$ is composed of volcanic and metasedimentary rocks in the ratio of $3: 1$. The most common are metabasalts and their tuffs, less common - metaandesites, metatrachyandesites and metapicrites (Kola Superdeep, 1998). Metasedimentary rocks consist of phyllites, siltstones, carbonate rocks, sandstones, grits and conglomerates. Throughout the complex there are sills of gabbro, ultrabasic rocks and dacite porphyrite. The Pechenga complex is divided into Matert (9-1059 m), Zhdanov (1059-2805 m), Zapolyarny (2805-4673 m), Luchlompolo (4673-4844 m), Pirttijarvi (4844-5642 $\mathrm{m})$, Kuvernerinjoki (5642-5717 m), Majarvi (5717$6835 \mathrm{~m}$ ) and Televi (6835-6842 m) formations (Orlov and Laverov, 1998).

The Archaean complex is represented by unit I composed predominantly of schists, gneisses with high-alumina minerals - HAM and amphibolites (6842-7622 m), unit II of gneisses with high-calcium minerals - HCM and amphibolites (7622-9456 m), unit III of gneisses with HAM and amphibolites (9456-9562 m), unit IV of gneisses with HCM, amphibolites and schists (9562-10144 m), unit $\mathrm{V}$ of 


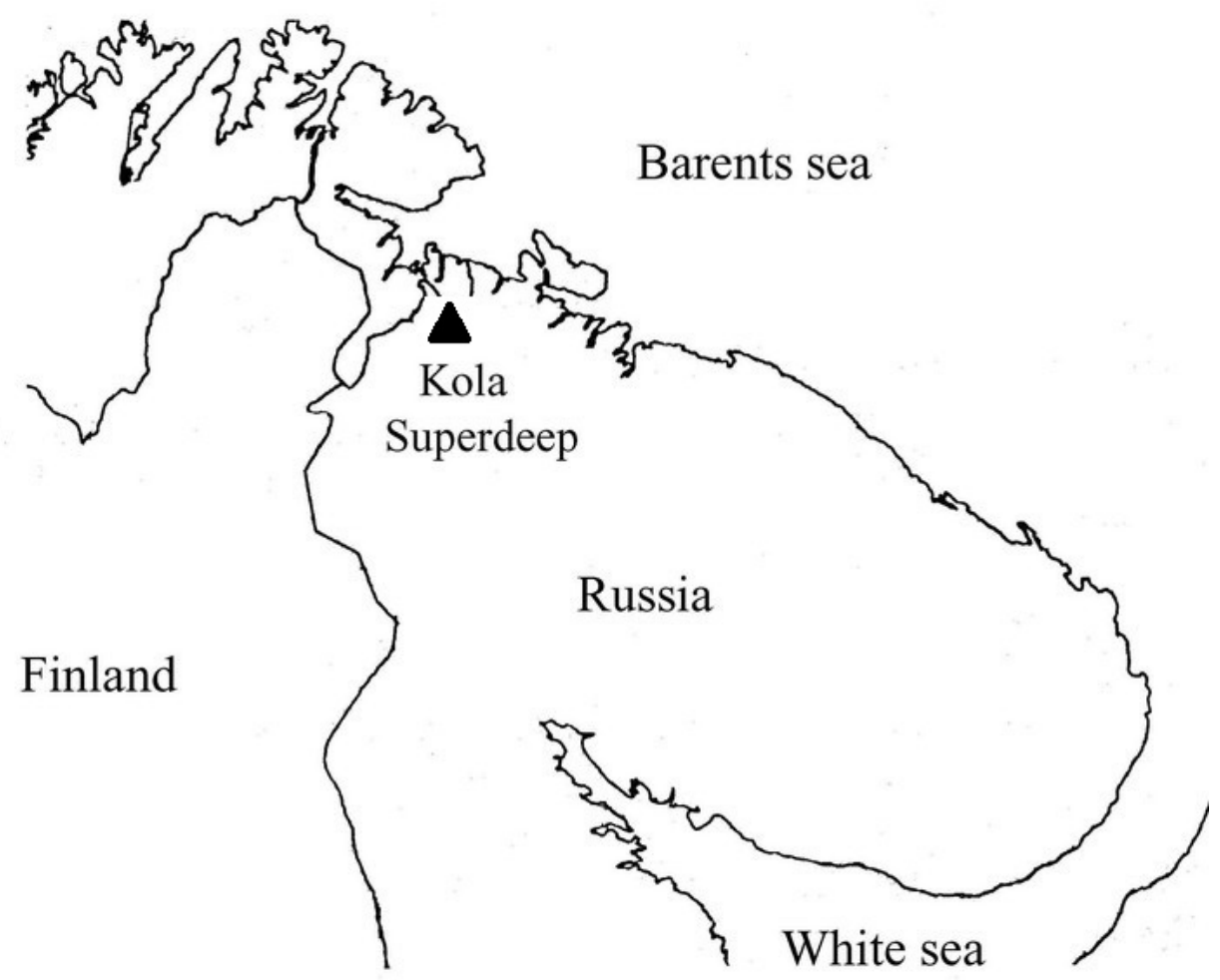

Fig. 1 Geographical location of the Kola Superdeep Borehole.

gneisses with HAM and schists (10144-10278 m), unit VI of gneisses with HCM, schists and amphibolite (10278-10448 m), unit VII of gneisses with HAM schists and amphibolites (10448-10601 m), unit VIII of gneisses and amphibolites (10601-11411 m), unit IX of gneisses with HAM, amphibolites and schists (11411-11708 m), unit X of biotite-plagioclase gneissses with HCM, amphibolite, schists and magnetite dissemination (11708-12262 m) (Structure, 2015).

As follows from the above data, the SG-3 section is represented by a very wide range of rocks from ultrabasic to acidic composition of different degree of processing, metamorphism, structural and textural features. This suggests possible wide variations of physical properties of constituent rocks. Rocks of prehnite-pumpellyite, greenschist and epidoteamphibolite facies occurring at depths of 9-6000 $\mathrm{m}$ in the Kola superdeep borehole section have a common origin and, in general, they do not differ much from the rocks selected at the outcrops elsewhere in the Pechenga complex (Glebovitsky, 1973; Petrov, 1999; Gorbatsevich and Trishina, 2017). Comparative characteristics of physical properties of rocks from the Archaean complex occurring deeper than 6842 metres are of particular interest. The expected data can provide a basis for estimating the degree of reflectionpassage of seismic waves at the interfaces of the predominant rocks in the vicinity of SG-3 and facilitate the correct interpretation of seismic profiles obtained from the earth's surface.

\section{METHODS OF INVESTIGATION}

The petrographic description of rocks and determination of their mineral composition was performed using their thin sections. For petrophysical measurements the samples were prepared in the form of a cube with the edge of $2.5-3 \mathrm{~cm}$ (Fig. 2). The rock density was determined by the Archimedes method. Compression and shear waves velocities were determined by the acoustopolarization method (Gorbatsevich, 1999 and 2009) using the device acoustopolariscope (Patent № 1281993, USSR, 1987).

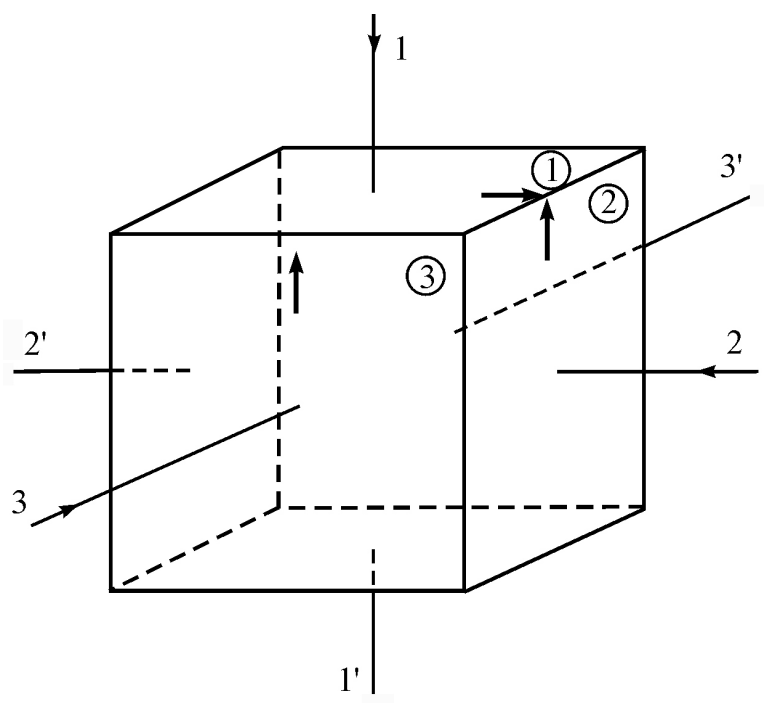

Fig. 2 Sketch of a cubic sample and marking its axes. 
The device comprises a radiator and receiver of purely shear linearly polarized ultrasonic waves (Patent No. 785737, USSR, 1980), a goniometer and indicator of the platform rotation angles.

The acoustopolariscope transducers are connected to the ultrasonic flaw detector. The measurements were carried out at the device operating frequency of 1.2 MHz. Before the measurements the sample was placed on the rotating platform of the acoustopolariscope. A well-conducting shear waves coupling medium was applied to the working surfaces of the radiator and receiver. During the measurements the electric drive rotated the platform within the full rotation angle of $360^{\circ}$ (Kovalevskiy, 2009). The envelope amplitude of the passing wave pulse was fixed on the screen of the ultrasonic device.

At the first stage the measurements are made at parallel polarization vectors (PVs) of the source and receiver (VP position). The amplitudes of the waves that have passed through the sample are measured. At the second stage the PVs of the transducers are set at a right angle (VC position). The measurement results are VP and VC acoustopolarigrams - circle diagrams of changes in the amplitude of the pulse envelope within the full rotation angle of the rotating platform. The measurements are made on all three pairs of faces of the cubic sample, Figure 2.

Pleochroism is observed as the light passes through transparent media (Shurkliff, 1962; Berry et al., 1983). It reflects an ability to absorb a beam of polarized light in various ways when rotating its polarization vector with respect to the medium structural elements. In this case, as a rule, the largest absorption of the polarized light is observed when the PVs are directed perpendicularly to linearly stretched structural elements of the medium. It is known that rocks, especially metamorphic, often have directed structure (Allison and Palmer, 1980). In this case, by analogy with the optical phenomenon pleochroism, one can observe the phenomenon of LAAA - the effect of linear acoustic anisotropic absorption during propagation of shear waves (Gorbatsevich, 2009). Depending on the absorption degree the VP acoustopolarigrams acquire the elongated shape. The presence and manifestation degree of the LAAA effect is determined by the VP acoustopolarigrams (Gorbatsevich, 2009). The manifestation degree of the LAAA effect is estimated by the formula:

$$
D=\frac{A_{R E}-A_{R R}}{A_{R E}+A_{R R}},
$$

where $A_{R E}$ and $A_{R R}$ are the amplitudes of the highest and lowest wave transmission (the largest and smallest diameters of the VP acoustopolarigrams).

The acoustopolarigrams obtained at the $\mathrm{VC}$ position allow one to determine the number and orientation of the projections of elastic symmetry elements of the anisotropic sample. The orientation of the elastic symmetry elements of the projections on the three faces of the cube were determined from the VC acoustopolarigrams. These projections are the directions, in which the compression and shear wave velocities take extreme values. An extended description of the acoustopolariscopy method is given in (Gorbatsevich, 2009).

The measurement results of the compression $\left(V_{\mathrm{P}}\right)$ and shear $\left(V_{\mathrm{S}}\right)$ wave velocities for all the faces of the cubic sample are determined in the form of a quasimatrix (Gorbatsevich et al., 1997),

$V_{\mathrm{ij}}=\begin{array}{lll}V_{11} & V_{12} & V_{13} \\ V_{21} & V_{22} & V_{23} \\ V_{31} & V_{32} & V_{33}\end{array}$,

where $V_{11}, V_{22}, V_{33}$ are compression wave velocities measured in the directions 1-1', 2-2', 3-3'; $V_{12}$ and $V_{13}$ are shear wave velocities measured in the direction 1-1' with PVs in the directions 2-2', 3-3'; $V_{21}, V_{23}-$ in the direction 2-2' with the PVs of the shear wave in the directions 1-1', 3-3'; $V_{31}, V_{32}-$ in the direction 3-3' with the PVs in the directions 1-1', 2-2', respectively (Fig. 2).

The anisotropy factors were calculated by the formula

$$
\begin{aligned}
A_{P}= & \frac{1}{V_{P R}} . \\
& \cdot \sqrt{\left(V_{11}-V_{A V}\right)^{2}+\left(V_{22}-V_{A V}\right)^{2}+\left(V_{33}-V_{A V}\right)^{2}},
\end{aligned}
$$

where $V_{P R}=\left(V_{11}+V_{22}+V_{33}\right) / 3$ is the mean value of compression wave velocity in the anisotropic sample.

To assess the anisotropy degree of the sample from the shear wave velocity the generalized anisotropy factor $B_{S}$ was calculated by the formula:

$$
B_{S}=\sqrt{B_{1}^{2}+B_{2}^{2}+B_{3}^{2}},
$$

$$
\text { where } \begin{aligned}
B_{1} & =\frac{2 \cdot\left(V_{12}-V_{13}\right)}{\left(V_{12}+V_{13}\right)} ; B_{2}=\frac{2 \cdot\left(V_{21}-V_{23}\right)}{\left(V_{21}+V_{23}\right)} ; \\
B_{3} & =\frac{2 \cdot\left(V_{31}-V_{32}\right)}{\left(V_{31}+V_{32}\right)}
\end{aligned}
$$

are the factors of shear-wave splitting (Crampin, 1985), defined for the directions 1-1', 2-2', 3-3', respectively.

The mean values for the shear wave velocities of the sample were calculated as

$V_{\mathrm{SR}}=\left(V_{12}+V_{13}+V_{21}+V_{23}+V_{31}+V_{32}\right) / 6$.

Because of weathering and other influence factors, rock samples usually collected on the Earth surface have lower density and velocity characteristics $\left(\rho, V_{\mathrm{P}}, V \mathrm{~s}\right)$, than those in a depth range of $0.1-3 \mathrm{~km}$ and deeper (Handbook 1975; Kern and Schmidt, 1990; Kern et al., 2001). A decrease in these characteristics also occurs during stress release (Gorbatsevich, 2003). 
This depth range is most interesting for exploration geophysics. Several studies have shown that the differences in the values of $\rho, V_{\mathrm{P}}$ and $V_{\mathrm{S}}$ at great depths are quite close to those determined by their mineral composition (Aleksandrov and Ryzhova, 1961; Belikov et al., 1970; Christensen and Mooney, 1995; Rudnick and Fountain, 1995; Berckhemer et al., 2000; Golovataya et al., 2006; Kern et al., 2009). Therefore, the values of density and velocity of compression and shear waves were calculated by the rock mineral composition. The calculated values of these velocities considering the specific mineral composition of the rock were taken as initial. The calculations were done by the formula (Belikov et al., 1970):

$$
\ln V_{k}=\frac{\Sigma P_{i} \ln \left(V_{i}\right)}{\Sigma P_{i}}
$$

where $V_{k}$ is the average calculated velocity in the rock, $V_{i}$ - the average velocity in each mineral, $P_{i}-$ the mineral share in the rock. The densities of the same samples were calculated by a similar formula. The rock mineral composition and corresponding velocity mean values in each particular mineral were taken as initial data (Belikov et al., 1970, Kern et al., 2009).

On the basis of density and velocity characteristics some technical constants were calculated: elasticity modulus $E$, shear modulus $G$ and Poisson's ratio $v$ by the following formulae:

$E=\left[\rho V_{\mathrm{S}}^{2}\left(3 V_{\mathrm{P}}^{2} / V_{\mathrm{S}}^{2}-4\right)\right] /\left(V_{\mathrm{P}}^{2} / V_{\mathrm{S}}^{2}-1\right)$,

$G=\rho V_{\mathrm{S}}^{2}$,

$v=\left(V_{\mathrm{P}}^{2} / V_{\mathrm{S}}^{2}-2\right) /\left(2 V_{\mathrm{P}}^{2} / V_{\mathrm{S}}^{2}-2\right)$.

For determining $E, G$ and $v$ under the Earth's surface conditions experimental $\rho_{\mathrm{R}}, V_{\mathrm{PR}}, V_{\mathrm{SR}}$ were used, under deep conditions the calculated $\rho_{\mathrm{C}}, V_{\mathrm{PC}}$, $V_{\mathrm{SC}}$ were used. The petrographic description of the rocks, values of density, compression and shear wave velocities, elastic moduli, Poisson's ratios for the surface and deep conditions are given in Tables 1-3.

\section{PETROGRAPHIC DESCRIPTION OF THE SAMPLES}

Seventeen core samples of the SG-3 main rocks from a depth range of 7263-11487 m were selected for determinations. The samples selected were gneisses, schists and amphibolites. Figure 3 shows the thin sections of rocks from the SG-3 Archaean section. The texture and mineral composition of the rocks are presented in Table 1. Gneisses have a medium-grained lepidogranoblastic texture. The main rock-forming minerals of gneisses (in \%) are plagioclase (47-65), biotite (11-42) and quartz (0.7-24). Garnet $\sim 12 \%$ and kyanite $\sim 8 \%$ are present in a less significant volume. Accessory minerals: ilmenite, epidote, zircon and apatite occupy a small fraction of the volume. The selected samples of schists have mainly mediumgrained, lepidogranoblastic texture. Schists contain

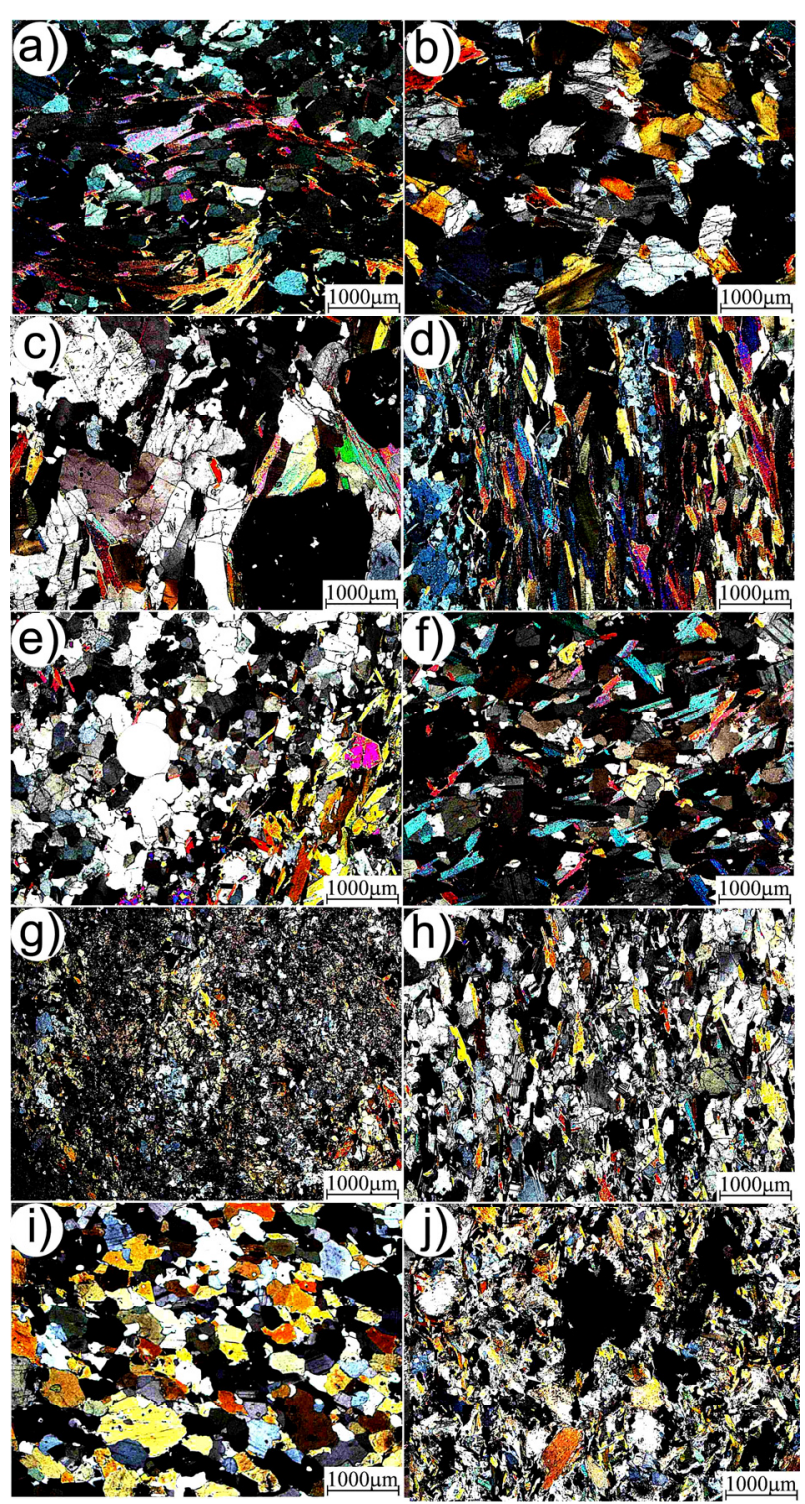

Fig. 3 The thin section micrographs of the main Archaean rocks from the section of the Kola superdeep borehole (SG-3). Gneisses: a) SG23881a, b) SG-41154-2, c) SG-42148-2. Schists: d) SG-23542n, e) SG-30025n, f) SG39164. Amphibolites: g) SG-23467, h) SG28186n, i) SG-40903n, j) SG-26158a.

(in \%): plagioclase (43-64), biotite (12-42), quartz (2$18)$, epidote $(\sim 12)$ and muscovite $(\sim 8)$. Accessory minerals are hornblende, ilmenite, apatite, chlorite, epidote, zircon and ore minerals. The texture of amphibolite rocks is mainly medium-grained, nematogranoblastic, schistose. The main rock-forming minerals of amphibolites are (in \%): hornblende (30$83)$, plagioclase (4-43), quartz ( 11). Apatite, epidote, ilmenite, biotite, chlorite, zircon and ore minerals are presented as accessory minerals.

\section{PHYSICAL PROPERTIES OF GNEISS, SCHIST AND AMPHIBOLITE}

Petrophysical properties of the samples are given in Table 2. It presents: experimentally measured $\left(\rho_{\mathrm{R}}\right)$ 
Table 1 Mineral composition and texture of rock samples from the SG-3 Archaean section.

\begin{tabular}{|c|c|c|c|c|}
\hline Sample № & Depth, $m$ & Mineral composition, $\%$ & Texture & Rock determination \\
\hline \multicolumn{5}{|c|}{ Gneisses } \\
\hline $\begin{array}{l}\text { SG-23881a } \\
\text { polished. }\end{array}$ & $\begin{array}{l}7382.1- \\
7396.1\end{array}$ & $\begin{array}{l}\text { Bt -13.3; Grt-6.5; Pl-54.9; } \\
\text { Qtz-23.7; Ilm-1.5; Ep-0.05; } \\
\text { Zrn-0.05 }\end{array}$ & $\mathrm{m} / \mathrm{g}$, lepidogranoblastic, porphyry & garnet-biotite gneiss \\
\hline SG-38631n & $\begin{array}{l}10502.0- \\
10518.6\end{array}$ & $\begin{array}{l}\text { Bt-34.6; Ms-5.5; Pl-47.3; } \\
\text { Qtz-10.4; Ilm-2.2; } \\
\text { sin. gr. Ap \& Zrn }\end{array}$ & $\begin{array}{c}\text { Inequigranular, lepidogranoblastic, } \\
\text { slight recrystallization, separations } \\
\text { of Qtz-Pl composition }\end{array}$ & muscovite-biotite gneiss \\
\hline SG-41154-2 & $\begin{array}{l}11324.0- \\
11336.0\end{array}$ & $\begin{array}{l}\text { Bt -42.3; Ky-8.4; Pl-46.9; } \\
\text { Qtz-0.7; Ilm-1.45; Ep-0.25; }\end{array}$ & $\mathrm{m} / \mathrm{g}$, lepidogranoblastic & $\begin{array}{l}\text { kyanite-biotite gneiss } \\
\text { aluminiferous }\end{array}$ \\
\hline SG-42003n & $\sim 11487.05$ & $\begin{array}{l}\text { Bt -14.0; Ky-5.3; Pl-65.4; } \\
\text { Qtz-4.1; Ilm-5.4; Ep-4.25; } \\
\text { Sil-1.5; Ap-0.05 }\end{array}$ & $\mathrm{m} / \mathrm{g}$, lepidogranoblastic & $\begin{array}{c}\text { kyanite-biotite gneiss } \\
\text { aluminiferous with sillimanite }\end{array}$ \\
\hline SG-42148-2 & $\sim 11487.05$ & $\begin{array}{l}\text { Grt-12.6; Bt -10.8; Ky-5.3; } \\
\text { Pl-57.4; Qtz-7.1; Ilm-2.4; } \\
\text { Sil-4.5 }\end{array}$ & $\mathrm{m} / \mathrm{g}$, lepidogranoblastic, porphyry & $\begin{array}{l}\text { garnet-kyanite-biotite gneiss } \\
\text { aluminiferous with sillimanite }\end{array}$ \\
\hline \multicolumn{5}{|c|}{ Schists } \\
\hline $\begin{array}{l}\text { SG-23542H } \\
\text { polished }\end{array}$ & $\begin{array}{l}7331.4- \\
7340.8\end{array}$ & $\begin{array}{c}\text { Bt -41.6; Ms-8.5; Pl-42.9; } \\
\text { Qtz-2.2; Hbl-0.1; Ttn-0.2; } \\
\text { Ilm-3.0; Ap-0.1; Chl-0.3. Ep- } \\
\text { 1.0; Zrn-0.1 }\end{array}$ & $\begin{array}{l}\mathrm{m} / \mathrm{g} \text {, lepidogranoblastic, intensive } \\
\text { shearing, layer-by-layer } \\
\text { recrystallization with formation of } \\
\text { Bt aggregates }\end{array}$ & two-mica schist \\
\hline SG-23696a & $\begin{array}{l}7357.6- \\
7366.6\end{array}$ & $\begin{array}{c}\text { Bt -18.4; Ms-8.3; Grt-1.5; Pl- } \\
\text { 49.9; Qtz-18.0; Ilm-1.6; Ap- } \\
\text { 0.05; Ep-2.2; Zrn-0.05 }\end{array}$ & $\begin{array}{l}\mathrm{m} / \mathrm{g} \text {, lepidogranoblastic, intensive } \\
\text { shearing, layer-by-layer } \\
\text { recrystallization with formation of } \\
\text { Qtz-Pl aggregates }\end{array}$ & $\begin{array}{l}\text { two-mica schist with } \\
\text { garnet }\end{array}$ \\
\hline SG-30025H & $\sim 8107.1$ & $\begin{array}{l}\text { Ep-12.4; Bt-23.9; P1-47.3; } \\
\text { Qtz-12.6; Or-3.5; Ttn-0.3 }\end{array}$ & $\begin{array}{l}\text { inequigranular, lepidogranoblastic, } \\
\text { porphyry, recrystallization, } \\
\text { separations of Or-Qtz-P1 } \\
\text { composition }\end{array}$ & Ep-Bt schist \\
\hline SG-34016H & $\sim 8865.95$ & $\begin{array}{l}\text { Bt -11.7; Ms-1.4; Ep-4.4; } \\
\text { Qtz-18.0; P1-64.4; Ttn-0.1 }\end{array}$ & $\begin{array}{l}\mathrm{m} / \mathrm{g} \text {, lepidogranoblastic, } \\
\text { recrystallization, separations of Qtz- } \\
\mathrm{Pl} \text { composition }\end{array}$ & $\begin{array}{l}\text { epidote-biotite schist with } \\
\text { muscovite }\end{array}$ \\
\hline SG-39164 & $\begin{array}{l}10666.8- \\
10679.0\end{array}$ & $\begin{array}{l}\text { Ep-8.6; Bt-28.3; Pl-44.2; Qtz- } \\
\text { 12.2; Chl-1.2; Ilm-2.2; Ttn- } \\
\text { 3.3; sin. gr. on Ap and Zrn }\end{array}$ & $\begin{array}{c}\text { inequigranular, lepidogranoblastic, } \\
\text { slight recrystallization, separations } \\
\text { of Qtz-Pl composition }\end{array}$ & epidote-biotite schist \\
\hline \multicolumn{5}{|c|}{ Amphibolites } \\
\hline $\begin{array}{l}\text { SG-23467 } \\
\text { polished }\end{array}$ & $\begin{array}{l}7263.0- \\
7275.1\end{array}$ & $\begin{array}{l}\text { Hbl-63.5; Pl-20.1; Qtz-5.1; } \\
\text { Ttn-0.3; Bt -1.6; Ilm-3.9; Or- } \\
\text { 3.4; Ap-0.4; Cb-1.7 }\end{array}$ & $\begin{array}{l}\mathrm{m} / \mathrm{g} \text {, nematogranoblastic, gabbro- } \\
\text { ophytic relics, lenticular isolations } \\
\text { of } \mathrm{Hb}-\mathrm{Qz}-\mathrm{Cb} \text { composition }\end{array}$ & $\begin{array}{c}\text { feldspar } \\
\text { amphibolite }\end{array}$ \\
\hline $\begin{array}{l}\text { SG-26158a } \\
\text { H. }\end{array}$ & $\sim 7695.25$ & $\begin{array}{l}\text { Hbl-83.3; Pl-4.1; Bt -3.6; } \\
\text { Ilm-4.2; Srp -4.4; Ep-0.4 }\end{array}$ & $\begin{array}{l}\mathrm{m} / \mathrm{g} \text {, granoblastic, relicts of } \\
\text { panidiomorphic grained }\end{array}$ & $\begin{array}{c}\text { anchimonomineral } \\
\text { amphibolite (metapyroxenite }\end{array}$ \\
\hline $\begin{array}{l}\text { SG-26977H } \\
\text { polished }\end{array}$ & $\begin{array}{l}7994.4- \\
8000.3\end{array}$ & $\begin{array}{c}\text { Hbl-46.8; Bt -11.1; Pl-22.1; } \\
\text { Qtz-0.2; Or-2.4; Ep-15.1; } \\
\text { Ttn-2.3 }\end{array}$ & $\begin{array}{c}\mathrm{m} / \mathrm{g} \text {, nematogranoblastic, strong } \\
\text { shearing }\end{array}$ & $\begin{array}{c}\text { feldspar } \\
\text { amphibolite }\end{array}$ \\
\hline SG-28186H & $\begin{array}{l}8213.9- \\
8222.0\end{array}$ & $\begin{array}{l}\text { Hbl -30.0; Bt-5.2; Pl-42.8; } \\
\text { Qtz-4.4; Or-3.0; Ep-14.3; } \\
\text { Ttn-0.3 }\end{array}$ & $\begin{array}{l}\mathrm{m} / \mathrm{g}, \text { nematogranoblastic, strong } \\
\text { shearing, recrystallization, } \\
\text { isolations of Or-Qtz-P1 composition }\end{array}$ & $\begin{array}{c}\text { feldspar } \\
\text { amphibolite }\end{array}$ \\
\hline SG-31093H & $\begin{array}{l}8701.2- \\
8715.7\end{array}$ & $\begin{array}{l}\text { Hbl-61.7; Bt-0.2; Pl-32.9; } \\
\text { Ilm-5.2 }\end{array}$ & $\begin{array}{l}\mathrm{m} / \mathrm{g}, \text { nematogranoblastic, strong } \\
\text { shearing, recrystallization, } \\
\text { isolations with formation of } \mathrm{c} / \mathrm{g} \\
\text { aggregates of } \mathrm{Hbl} \text { and lenticular } \\
\text { aggregates of Ilm grains }\end{array}$ & $\begin{array}{c}\text { feldspar } \\
\text { amphibolite }\end{array}$ \\
\hline SG-37263 & $\sim 10253.7$ & $\begin{array}{c}\text { Hbl-60.57; Pl-20.0; Qtz-11.7; } \\
\text { Or-2.13; Ep-3.33; Chl-0.02; } \\
\text { Ilm-2.2; Ap-0.03; } \\
\text { Zrn-0.02 } \\
\end{array}$ & $\begin{array}{c}\mathrm{m} / \mathrm{g}, \text { nematogranoblastic, slight } \\
\text { shearing }\end{array}$ & $\begin{array}{c}\text { feldspar } \\
\text { amphibolite }\end{array}$ \\
\hline SG-40903н & $\begin{array}{l}11253.7- \\
11263.0\end{array}$ & $\begin{array}{l}\text { Hbl-66.9; Pl-22.1; Qtz-7; } \\
\text { Ep-0.35; Ilm-3.6; Ap-0.05 }\end{array}$ & $\begin{array}{c}\mathrm{m} / \mathrm{g}, \text { nematogranoblastic, slight } \\
\text { shearing }\end{array}$ & $\begin{array}{c}\text { feldspar } \\
\text { amphibolite }\end{array}$ \\
\hline
\end{tabular}

Note. The symbols are as in (Kretz, 1983). 
Table 2 Petrophysical properties of samples from the SG-3 Archaean section. Experimental and calculated data.

\begin{tabular}{|c|c|c|c|c|c|c|c|c|c|c|}
\hline Sample № & Rock name & $\begin{array}{l}\rho_{\mathrm{R}}, \\
\mathrm{g} / \mathrm{cm} 3\end{array}$ & $\begin{array}{l}\rho_{\mathrm{C}}, \\
\mathrm{g} / \mathrm{cm} 3\end{array}$ & $\begin{array}{l}\text { Velocity } \\
V_{\mathrm{ij}}, \mathrm{km} / \mathrm{s}\end{array}$ & matrix & $\begin{array}{l}V_{\mathrm{PR}} \\
\mathrm{km} / \mathrm{s}\end{array}$ & $\begin{array}{l}V_{\mathrm{PC}} \\
\mathrm{km} / \mathrm{s}\end{array}$ & $\begin{array}{l}V_{\mathrm{SR}} \\
\mathrm{km} / \mathrm{s}\end{array}$ & $\begin{array}{l}V_{\mathrm{SC}} \\
\mathrm{km} / \mathrm{s}\end{array}$ & $\begin{array}{l}\text { LAAA } \\
D_{1}, \\
D_{2}, \\
D_{3} \\
\end{array}$ \\
\hline \multicolumn{11}{|l|}{ Gneiss } \\
\hline $\begin{array}{l}\text { SG-23881a } \\
\text { polished }\end{array}$ & Garnet-biotite gneiss & 2.68 & 2.82 & $\begin{array}{ll}5.87 & 2.47 \\
2.54 & 5.62 \\
1.97 & 1.94 \\
\end{array}$ & $\begin{array}{l}3.11 \\
2.80 \\
4.24 \\
\end{array}$ & 5.24 & 6.33 & 2.47 & 3.64 & $\begin{array}{l}0.53 \\
0.70 \\
0.0 \\
\end{array}$ \\
\hline SG-38631n & $\begin{array}{l}\text { Muscovite- biotite } \\
\text { gneiss }\end{array}$ & 2.50 & 2.81 & $\begin{array}{cc}3.36 & 2.42 \\
2.59 & 3.28 \\
--^{-} & --^{6} \\
\end{array}$ & $\begin{array}{l}2.19 \\
2.53 \\
3.06 \\
\end{array}$ & 3.23 & 6.15 & 2.43 & 3.35 & $\begin{array}{l}0.29 \\
0.01 \\
0.20 \\
\end{array}$ \\
\hline SG-41154-2 & $\begin{array}{l}\text { Kyanite-biotite gneiss } \\
\text { aluminiferous }\end{array}$ & 2.97 & 2.92 & $\begin{array}{cc}3.87 & 2.36 \\
--\cdot & --‘ \\
--^{-} & --6 \\
\end{array}$ & $\begin{array}{c}2.19 \\
--{ }^{6} \\
--^{6}\end{array}$ & 3.87 & 6.39 & 2.28 & 3.38 & $\begin{array}{l}0.28 \\
0.45 \\
0.58 \\
\end{array}$ \\
\hline SG-42003n & $\begin{array}{l}\text { Kyanite-biotite gneiss } \\
\text { aluminiferous, with } \\
\text { sillimanite }\end{array}$ & 2.63 & 2.89 & $\begin{array}{cc}2.57 & 1.93 \\
1.96 & 1.33 \\
--^{-} & --{ }^{-}\end{array}$ & $\begin{array}{c}2.16 \\
1.91 \\
--\cdot\end{array}$ & 1.95 & 6.44 & 1.99 & 3.55 & $\begin{array}{l}0.62 \\
0.77 \\
0.02\end{array}$ \\
\hline SG-42148-2 & $\begin{array}{l}\text { Garnet-Kyanite-biotite } \\
\text { gneiss aluminiferous, } \\
\text { with sillimanite }\end{array}$ & 2.85 & 2.97 & $\begin{array}{cc}2.41 & 1.70 \\
1.43 & 2.95 \\
-- & --{ }^{-}\end{array}$ & $\begin{array}{r}1.48 \\
1.56 \\
---^{6}\end{array}$ & 2.68 & 6.63 & 1.54 & 3.70 & $\begin{array}{l}0.51 \\
0.29 \\
0.06\end{array}$ \\
\hline Average & & $\begin{array}{l}2.73 \\
\pm 0.17 \\
\end{array}$ & $\begin{array}{l}2.88 \\
\pm 0.06 \\
\end{array}$ & & & $\begin{array}{l}3.39 \\
\pm 1.11 \\
\end{array}$ & $\begin{array}{l}6.38 \\
\pm 0.16 \\
\end{array}$ & $\begin{array}{l}2.14 \\
\pm 0.34 \\
\end{array}$ & $\begin{array}{l}3.52 \\
\pm 0.14 \\
\end{array}$ & $\begin{array}{l}0.35 \\
\pm 0.26 \\
\end{array}$ \\
\hline \multicolumn{11}{|l|}{ Schist } \\
\hline $\begin{array}{l}\text { SG-23542n } \\
\text { polished }\end{array}$ & Two-mica schist & 2.89 & 2.85 & $\begin{array}{lc}1.40 & 1.83 \\
1.40 & 2.46 \\
4.64 & --\cdot \\
\end{array}$ & $\begin{array}{l}1.73 \\
2.44 \\
5.41 \\
\end{array}$ & 3.09 & 6.39 & 2.41 & 3.29 & $\begin{array}{l}0.72 \\
0.88 \\
0.54 \\
\end{array}$ \\
\hline SG-23696a & $\begin{array}{l}\text { Two-mica schist with } \\
\text { garnet }\end{array}$ & 2.67 & 2.76 & $\begin{array}{ll}4.76 & 2.30 \\
2.53 & 4.15 \\
2.60 & 2.65 \\
\end{array}$ & $\begin{array}{l}2.90 \\
2.09 \\
5.89\end{array}$ & 4.93 & 6.44 & 2.50 & 3.51 & $\begin{array}{l}0.25 \\
0.65 \\
0.34 \\
\end{array}$ \\
\hline SG-30025n & Ep-Bt schist & 2.53 & 2.83 & $\begin{array}{ll}4.72 & 2.33 \\
2.06 & 2.65 \\
1.68 & 1.67 \\
\end{array}$ & $\begin{array}{l}2.02 \\
1.76 \\
1.91 \\
\end{array}$ & 3.09 & 6.63 & 1.92 & 3.50 & $\begin{array}{l}0.18 \\
0.58 \\
0.58 \\
\end{array}$ \\
\hline SG-34016n & $\begin{array}{l}\text { Epidote-biotite schist } \\
\text { with muscovite }\end{array}$ & 2.59 & 2.73 & $\begin{array}{ll}5.51 & 3.05 \\
2.50 & 4.29 \\
1.94 & 2.01 \\
\end{array}$ & $\begin{array}{l}2.51 \\
2.21 \\
4.29 \\
\end{array}$ & 4.70 & 6.25 & 2.37 & 3.53 & $\begin{array}{l}0.43 \\
0.66 \\
0.69 \\
\end{array}$ \\
\hline SG-39164 & Epidote-biotite schist & 2.78 & 2.93 & $\begin{array}{ll}4.81 & 2.57 \\
2.00 & 3.14 \\
1.48 & 1.36 \\
\end{array}$ & $\begin{array}{c}2.31 \\
1.98 \\
--^{6}\end{array}$ & 3.98 & 6.31 & 1.95 & 3.48 & $\begin{array}{l}0.40 \\
0.75 \\
0.75 \\
\end{array}$ \\
\hline Average & & $\begin{array}{l}2.69 \\
\pm 0.13 \\
\end{array}$ & $\begin{array}{l}2.82 \\
\pm 0.07 \\
\end{array}$ & & & $\begin{array}{l}3.96 \\
\pm 0.77 \\
\end{array}$ & $\begin{array}{l}6.40 \\
\pm 0.13 \\
\end{array}$ & $\begin{array}{l}2.23 \\
\pm 0.24 \\
\end{array}$ & $\begin{array}{l}3.46 \\
\pm 0.09 \\
\end{array}$ & $\begin{array}{l}0.56 \\
\pm 0.20 \\
\end{array}$ \\
\hline \multicolumn{11}{|l|}{ Amphibolite } \\
\hline $\begin{array}{l}\text { SG-23467 } \\
\text { polished }\end{array}$ & $\begin{array}{l}\text { Amphibolite } \\
\text { feldspathic }\end{array}$ & 2.80 & 3.06 & $\begin{array}{ll}6.81 & 4.12 \\
3.77 & 6.56 \\
3.65 & 3.10 \\
\end{array}$ & $\begin{array}{l}3.91 \\
3.78 \\
6.44 \\
\end{array}$ & 6.60 & 6.77 & 3.72 & 3.81 & $\begin{array}{l}0.05 \\
0.03 \\
0.13 \\
\end{array}$ \\
\hline $\begin{array}{l}\text { SG-26158a } \\
\text { н. }\end{array}$ & $\begin{array}{l}\text { Amphibolite } \\
\text { anchimonomineral } \\
\text { (metapyroxenite) }\end{array}$ & 3.08 & 3.18 & $\begin{array}{ll}5.80 & 3.76 \\
2.50 & 3.26 \\
2.05 & 2.39 \\
\end{array}$ & $\begin{array}{l}2.68 \\
2.24 \\
3.97 \\
\end{array}$ & 4.34 & 7.04 & 2.56 & 3.89 & $\begin{array}{l}0.29 \\
0.48 \\
0.39 \\
\end{array}$ \\
\hline $\begin{array}{l}\text { SG-26977n } \\
\text { polished }\end{array}$ & $\begin{array}{l}\text { Amphibolite } \\
\text { feldspathic }\end{array}$ & 2.96 & 3.09 & $\begin{array}{ll}5.77 & 2.58 \\
2.79 & 3.75 \\
1.84 & 1.68 \\
\end{array}$ & $\begin{array}{l}2.61 \\
2.36 \\
2.34 \\
\end{array}$ & 3.95 & 6.85 & 2.31 & 3.77 & $\begin{array}{l}0.02 \\
0.0 \\
0.07 \\
\end{array}$ \\
\hline SG-28186n & $\begin{array}{l}\text { Amphibolite } \\
\text { feldspathic }\end{array}$ & 2.87 & 2.87 & $\begin{array}{ll}2.57 & 2.40 \\
2.44 & 4.55 \\
1.71 & 2.38 \\
\end{array}$ & $\begin{array}{l}2.25 \\
2.62 \\
3.26 \\
\end{array}$ & 3.46 & 6.59 & 2.30 & 3.65 & $\begin{array}{l}0.08 \\
0.29 \\
0.59 \\
\end{array}$ \\
\hline SG-31093n & $\begin{array}{l}\text { Amphibolite } \\
\text { feldspathic }\end{array}$ & 2.93 & 3.07 & $\begin{array}{ll}5.96 & 2.21 \\
2.28 & 4.83 \\
2.58 & 2.62\end{array}$ & $\begin{array}{l}2.53 \\
2.68 \\
6.20\end{array}$ & 5.66 & 6.87 & 2.48 & 3.82 & $\begin{array}{l}0.55 \\
0.16 \\
0.0\end{array}$ \\
\hline SG-37263 & $\begin{array}{l}\text { Amphibolite } \\
\text { feldspathic }\end{array}$ & 2.93 & 3.02 & $\begin{array}{ll}5.22 & 2.69 \\
2.91 & 7.18 \\
2.42 & 2.66 \\
\end{array}$ & $\begin{array}{l}2.58 \\
4.14 \\
5.47 \\
\end{array}$ & 5.96 & 6.84 & 2.90 & 3.89 & $\begin{array}{l}0.09 \\
0.07 \\
0.14 \\
\end{array}$ \\
\hline SG-40903n & $\begin{array}{l}\text { Amphibolite } \\
\text { feldspathic }\end{array}$ & 2.97 & 3.06 & $\begin{array}{ll}3.55 & 2.77 \\
1.87 & 3.00 \\
1.65 & 1.69 \\
\end{array}$ & $\begin{array}{l}2.71 \\
1.89 \\
2.33 \\
\end{array}$ & 2.96 & 6.89 & 2.10 & 3.88 & $\begin{array}{l}0.07 \\
0.08 \\
0.05 \\
\end{array}$ \\
\hline Average & & $\begin{array}{l}2.93 \\
\pm 0.08\end{array}$ & $\begin{array}{l}3.05 \\
\pm 0.09 \\
\end{array}$ & & & $\begin{array}{l}4.70 \\
\pm 1.27 \\
\end{array}$ & $\begin{array}{l}6.84 \\
\pm 0.13 \\
\end{array}$ & $\begin{array}{l}2.62 \\
\pm 0.50 \\
\end{array}$ & $\begin{array}{l}3.82 \\
\pm 0.08\end{array}$ & $\begin{array}{l}0.17 \\
\pm 0.18 \\
\end{array}$ \\
\hline
\end{tabular}


Table 3 Moduli of elasticity and shearing and Poisson's ratios of the samples from the SG-3 Archaean section.

\begin{tabular}{|c|c|c|c|c|}
\hline \multirow{2}{*}{ Sample № } & \multirow{2}{*}{ Rock name } & \multicolumn{3}{|c|}{ Deep conditions } \\
\hline & & $E \cdot 10^{-4}, \mathrm{MPa}$ & $G \cdot 10^{-4}, \mathrm{MPa}$ & $v$ \\
\hline \multicolumn{5}{|c|}{ Gneiss } \\
\hline $\begin{array}{l}\text { SG-23881a } \\
\text { polished. }\end{array}$ & Garnet-biotite gneiss & 9.35 & 3.73 & 0.252 \\
\hline SG-38631n & Muscovite- biotite gneiss & 8.14 & 3.16 & 0.288 \\
\hline SG-41154-2 & Kyanite- biotite gneiss aluminiferous & 8.73 & 3.34 & 0.306 \\
\hline SG-42003n & $\begin{array}{l}\text { Kyanite- biotite gneiss aluminiferous, with } \\
\text { sillimanite }\end{array}$ & 9.34 & 3.64 & 0.282 \\
\hline SG-42148-2 & $\begin{array}{l}\text { Garnet-kyanite- biotite gneiss aluminiferous, with } \\
\text { sillimanite }\end{array}$ & 10.36 & 4.07 & 0.273 \\
\hline & Average & $9.18 \pm 0.74$ & $3.60 \pm 0.32$ & $0.290 \pm 0.020$ \\
\hline \multicolumn{5}{|c|}{ Schist } \\
\hline $\begin{array}{l}\text { SG-23542n } \\
\text { polished. }\end{array}$ & Two-mica schist & 8.02 & 3.09 & 0.300 \\
\hline SG-23696a & Two-mica schist with garnet & 8.60 & 3.40 & 0.265 \\
\hline SG-30025n & Ep-Bt schist & 8.85 & 3.46 & 0.278 \\
\hline SG-34016n & Epidote-biotite schist with muscovite & 8.62 & 3.41 & 0.265 \\
\hline SG-39164 & Epidote-biotite schist & 9.11 & 3.56 & 0.281 \\
\hline & Average & $8.65 \pm 0.37$ & $3.39 \pm 0.16$ & $0.280 \pm 0.020$ \\
\hline \multicolumn{5}{|c|}{ Amphibolite } \\
\hline SG-23467 polished. & Feldspathic amphibolite & 11.27 & 4.44 & 0.268 \\
\hline SG-26158a & Anchimonomineral amphibolite (metapyroxenite) & 12.32 & 4.81 & 0.280 \\
\hline $\begin{array}{l}\text { SG-26977n } \\
\text { polished. }\end{array}$ & Feldspathic amphibolite & 11.24 & 4.38 & 0.284 \\
\hline SG-28186n & Feldspathic amphibolite & 9.78 & 3.82 & 0.279 \\
\hline SG-31093n & Feldspathic amphibolite & 11.41 & 4.47 & 0.277 \\
\hline SG-37263 & Feldspathic amphibolite & 11.53 & 4.57 & 0.261 \\
\hline SG-40903n & Feldspathic amphibolite & 11.72 & 4.62 & 0.267 \\
\hline & Average & $11.33 \pm 0.72$ & $4.45 \pm 0.29$ & $0.280 \pm 0.017$ \\
\hline
\end{tabular}

and calculated by mineral composition $\left(\rho_{\mathrm{C}}\right)$ densities; matrix velocity $V_{\mathrm{ij}}$, average velocities of experimentally determined compression $\left(V_{\mathrm{PR}}\right)$ and shear $\left(V_{\mathrm{SR}}\right)$ waves. The Table also shows the calculated velocities of compression $\left(V_{\mathrm{PC}}\right)$ and shear $\left(V_{\mathrm{SC}}\right)$ waves and the LAAA index $D$. In a separate Table 3, dynamic elastic moduli and Poisson's ratios of rocks are presented.

A comparison of petrophysical properties of the main rock samples from the SG-3 Archaean section (Table 2) shows that the density obtained in lab conditions $\left(\rho_{\mathrm{R}}\right)$ is somewhat less than that calculated by mineral composition $\left(\rho_{\mathrm{C}}\right)$ for gneisses, schists and amphibolites. This is due to the fracture porosity formed as a result of lithostatic stress relief of deep samples.

A preliminary analysis of the samples elastic properties can be performed according to the outlines of the acoustopolarigrams (Fig. 4). All acoustopolarigrams show that rocks from the SG-3 Archaean section were under complex heterogeneous stress. Note that the VC diagrams of most of the samples have the shape of four-petalled figures, which indicates the presence of elastic anisotropy in them. The minima of the VC diagrams allow one to determine the spatial position of the symmetry elements (Gorbatsevich et al., 1997).
The acoustopolarigrams of the gneiss samples SG-23881a, SG-41154-2 and SG-42148-2 are characterized by pronounced manifestation of the LAAA effect on all faces with high LAAA indices. For the SG-23881a sample, index $D_{1}=0.53$, $D_{2}=0.70$. For the SG-41154-2, SG-42148-2 samples, the $D$ values are in the range of $0.28-0.58$. The photos of the thin sections show that the mineral grains of these samples are elongated in shape and oriented in one direction, which explains the presence of the LAAA effect. In the photo of the SG-42148-2 sample, there is a large inclusion, which probably affected the broken shape of the VP diagram obtained for the 3rd face. It should be noted that the thin section of this sample is made only in one section and does not reflect the volume structure of the rock.

The VP acoustopolarigrams of the schist samples (SG-23542n, SG-30025n and SG-39164) show an even more significant effect of linear acoustic anisotropic absorption. This is confirmed by very high indices of LAAA. For the sample SG-23542n, $D_{1}, D_{2}$ and $D_{3}$ are $0.72,0.88$ and 0.54 , respectively. For the SG-39164 sample, these values are $0.40,0.75$ and 0.75. Accordingly, in the photo of the schist thin sections, a more strict orientation of the grains stretched in one direction is noted than in the gneiss. It should be noted that the LAAA effect greatly reflects 


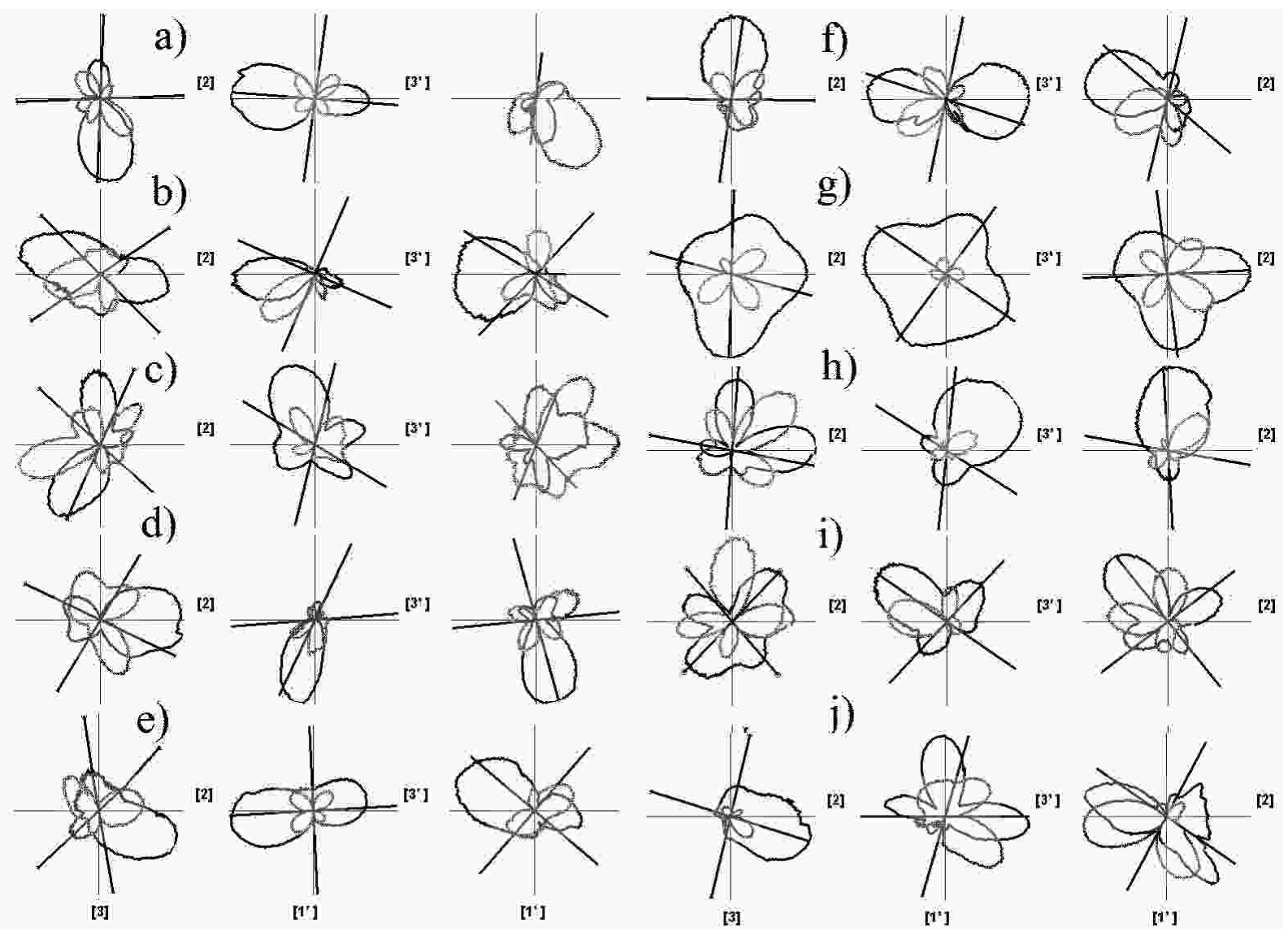

Fig. 4 Examples of acoustopolarigrams of the main rocks from the SG-3 Archaean section. Gneisses: a) SG-23881a, b) SG-41154-2, c) SG-42148-2. Schists: d) SG-23542n, e) SG-30025n, f) SG39164. Amphibolites: g) SG-23467, h) SG-28186n, i) SG-40903n, j) SG-26158a. Dark line -vectors parallel (VP); light line - vectors crossed (VC).

the acoustic properties contrast at the contacts between the neighboring mineral grains oriented in one direction and microcracks that are present at these contacts.

The VP acoustopolarigrams of the first two faces (1-1' and 2-2') of the SG-23467 amphibolite sample indicate the presence of moderate anisotropy, practically without the influence of LAAA, Figure 4. The same is reflected in the $D$ indices. On all three faces, the minima of the VC diagrams are observed. From the review of the thin section photo it follows that the directive orientation of the mineral grain forms is observed. Analysis of the acoustopolarigrams of the amphibolite sample SG-28186n indicates the presence of the LAAA effect on virtually all faces. For the sample SG-28186n, the values of $D_{1}=0.08$, $D_{2}=0.29$ and $D_{3}=0.59$ are quite significant. In the photo of the SG-28186n thin sections, the direction of the mineral grains is well traced.

A comparison of the LAAA average parameters for the rock as a whole shows that this index numerically reflects the orientation degree in the rock structure. For example, for schists, the oriented structure of which is most pronounced, $D_{\mathrm{sc}}=0.56$ \pm 0.20 . For gneisses, $D_{\mathrm{gn}}=0.35 \pm 0.26$. For amphibolites, which contain the smallest amount of mica, $D_{\text {am }}=0.17 \pm 0.18$.

In experimental determinations of the velocities measured in the samples under lab conditions, the complete quasi-matrix $V_{\mathrm{ij}}$ was not obtained for all samples. Because of overdamping of ultrasonic waves in some samples, the velocities were obtained in two or one direction. Incomplete velocity matrices were obtained for samples SG-23542n, SG-38631n, SG39164, SG-41154-2, SG-42003n and SG-42148-2. Moreover, the measured velocities have unusually low values that are not characteristic of hard rocks (Handbook, 1975; Christensen and Mooney, 1995). Variations in the changes of the mean values of compression wave velocities for gneisses diverge in a wide range $\left(V_{\mathrm{PR}}=1.95-5.24 \mathrm{~km} / \mathrm{s}\right)$. The same scatter is observed for the shear wave velocities, $V_{\mathrm{SR}}=1.54-$ $2.47 \mathrm{~km} / \mathrm{s}$. The average value of the experimentally measured compression velocities for gneisses is 3.39 $\pm 1.11 \mathrm{~km} / \mathrm{s}$, of the shear velocities $-2.14 \pm 0.34 \mathrm{~km} / \mathrm{s}$. Wide limits of the experimental velocities scatter are observed in schists and amphibolites. Such low values of the average compression and shear velocities and 
their wide scatter are not typical for gneisses, schists, amphibolites and other rocks in-situ composing the SG-3 section. The geophysical observations at depths of 7-12 km (deep seismic profiling, acoustic logging) show other data. The average compression velocities for this interval are close to $6.2 \mathrm{~km} / \mathrm{s}$, shear velocities - 3.6 km/s (Kozlowsky, 1987; Kola Superdeep, 1998).

As follows from the experimental results, the decompaction of rocks extracted from great depths has a significant effect on their velocity characteristics. In the SG-3 Archaean section, practically linear dependence of the compression wave velocity $V_{\mathrm{P}}$ in the samples of gneiss-granite rocks extracted from depths of 6905-12050 $\mathrm{m}$ has been revealed, Figure 5 (Gorbatsevich et al., 1997). This diagram shows that there is a fundamental limitation in the extraction depth of such rocks in a coherent shape (Gorbatsevich, 2003). Therefore, experimentally measured velocities reflect only one or another degree of this decompaction and are not suitable for calculating anisotropy factors.

The values of the velocity characteristics close to those for the rock in-situ conditions were obtained by calculation taking into account their mineral composition (formula (6)), Table 1 and 2. According to the calculations, the average values of the compression and shear wave characteristics calculated by mineral composition for gneisses vary within $V_{\mathrm{PC}}=6.15-6.63 \mathrm{~km} / \mathrm{s}$ and $V_{\mathrm{SC}}=3.36-3.70 \mathrm{~km} / \mathrm{s}$, respectively. The average compression velocity is $6.38 \pm 0.16 \mathrm{~km} / \mathrm{s}$, and the shear velocity is 3.52 $\pm 0.14 \mathrm{~km} / \mathrm{s}$. For schists, the variations in velocity characteristics are $V_{\mathrm{PC}}=6.25-6.63 \mathrm{~km} / \mathrm{s}$ and $V_{\mathrm{SC}}=$ $3.29-3.53 \mathrm{~km} / \mathrm{s}$. The average compression velocity is $6.40 \pm 0.13 \mathrm{~km} / \mathrm{s}$, the shear velocity is $3.46 \pm 0.09$ $\mathrm{km} / \mathrm{s}$. The interval of variation in the mean values of compression and shear wave characteristics for amphibolites is $V_{\mathrm{PC}}=6.59-7.04 \mathrm{~km} / \mathrm{s}$ and $V_{\mathrm{SC}}=3.65-$ $3.89 \mathrm{~km} / \mathrm{s}$. Their average is $6.84 \pm 0.13 \mathrm{~km} / \mathrm{s}$ and 3.82 $\pm 0.08 \mathrm{~km} / \mathrm{s}$, respectively.

According to the obtained averages, the highest velocities are observed for amphibolites, the lowest for gneisses and schists. A comparison of the average velocities of both compression and shear waves in gneisses and schists shows that there is a small difference between them. Since the calculation of the velocity values was made by the rock mineral composition, these averages reflect the influence of high-velocity amphibole in amphibolites and lowvelocity micas in schists. These data are close to the velocity values determined along the Kola superdeep borehole section by geophysical methods, including vertical seismic profiling and acoustic logging (Kozlowsky, 1987; Digranes et al., 1996; Kola Superdeep, 1998; Smithson et al., 2000; Trčková et al., 2002; The lithosphere ..., 2005). The difference between calculated and geophysical data can be explained by the presence of fluids in rocks in-situ. Taking into account the scatter, their values overlap. Gneisses, schists and amphibolites differ in the factor of elastic wave velocities. Thus, on the boundary between gneiss or schist and amphibolite, detectable reflections of a seismic wave are possible. The performed estimate disregards elastic anisotropy of the SG-3 rocks, which can reach significant values (Kern et al., 2001). At the same time, they provide a basis for assessing the possible degree of reflectionpassage of seismic waves at the interfaces of the predominant rocks in the vicinity of the SG-3 well.

The mean values of the moduli of elasticity $(E)$ and shearing $(G)$ and Poisson's ratios ( $v$ ) for deep conditions are given in Table 3 . The mean values of technical constants calculated by mineral composition - the Young's modulus $(E)$, the shearing modulus $(G)$ and Poisson's ratio $(v)$ are: for gneisses $(9.18 \pm 0.74)$ $10^{4} \mathrm{MPa},(3.60 \pm 0.32) 10^{4} \mathrm{MPa}, 0.290 \pm 0.020$; for schists $(8.65 \pm 0.37) 10^{4} \mathrm{MPa},(3.39 \pm 0.16) 10^{4} \mathrm{MPa}$, $v=0.280 \pm 0.020$; for amphibolites $(11.33 \pm 0.72) 10^{4}$ $\mathrm{MPa},(4.45 \pm 0.29) 10^{4} \mathrm{MPa}, v=0.280 \pm 0.017$, respectively The moduli relations in different rocks show the same tendencies as the average velocities. Their values are lower in schists, intermediate in gneisses, higher in amphibolites. These parameters can be used in assessing the stress state of rock masses at great depths.

\section{CONCLUSIONS}

Investigation of petrophysical properties of the Archaean section main rocks from the Kola superdeep borehole (SG-3) allow one to reveal the difference between gneisses, schists and amphibolites. Their main rock-forming minerals are plagioclase, hornblende, mica and quartz. The rock texture is mainly medium-grained, the structure is nematogranoblastic and lepidogranoblastic.

A survey of the acoustopolarigrams shows that most of the samples belong to elastic anisotropic media. The effect of linear acoustic anisotropic absorption (LAAA) is manifested, to some extent, in most samples. A comparison of the LAAA index for different rocks showed that this characteristics can assess numerically the orientation degree in the rock structure. The oriented structure in schists is most pronounced, it is least in amphibolites.

The density of the samples from the SG-3 Archaean section obtained under lab conditions is somewhat less than that calculated by mineral composition, and it is lower in schists and higher in amphibolites. Variations in the changes of the mean values of compression wave velocities measured experimentally for gneisses are $1.95-5.24 \mathrm{~km} / \mathrm{s}$, for shear wave velocities $-V_{\mathrm{SR}}=1.54-2.47 \mathrm{~km} / \mathrm{s}$. The wide limits of the experimental velocities scatter are observed in schists and amphibolites. The effect of decompaction has a significant influence on the compression and shear wave velocities in the samples extracted from depths of 7-12 km. The velocities in the samples have unusually low values that are not characteristic of hard solid rocks that occur at the depth of extraction. 


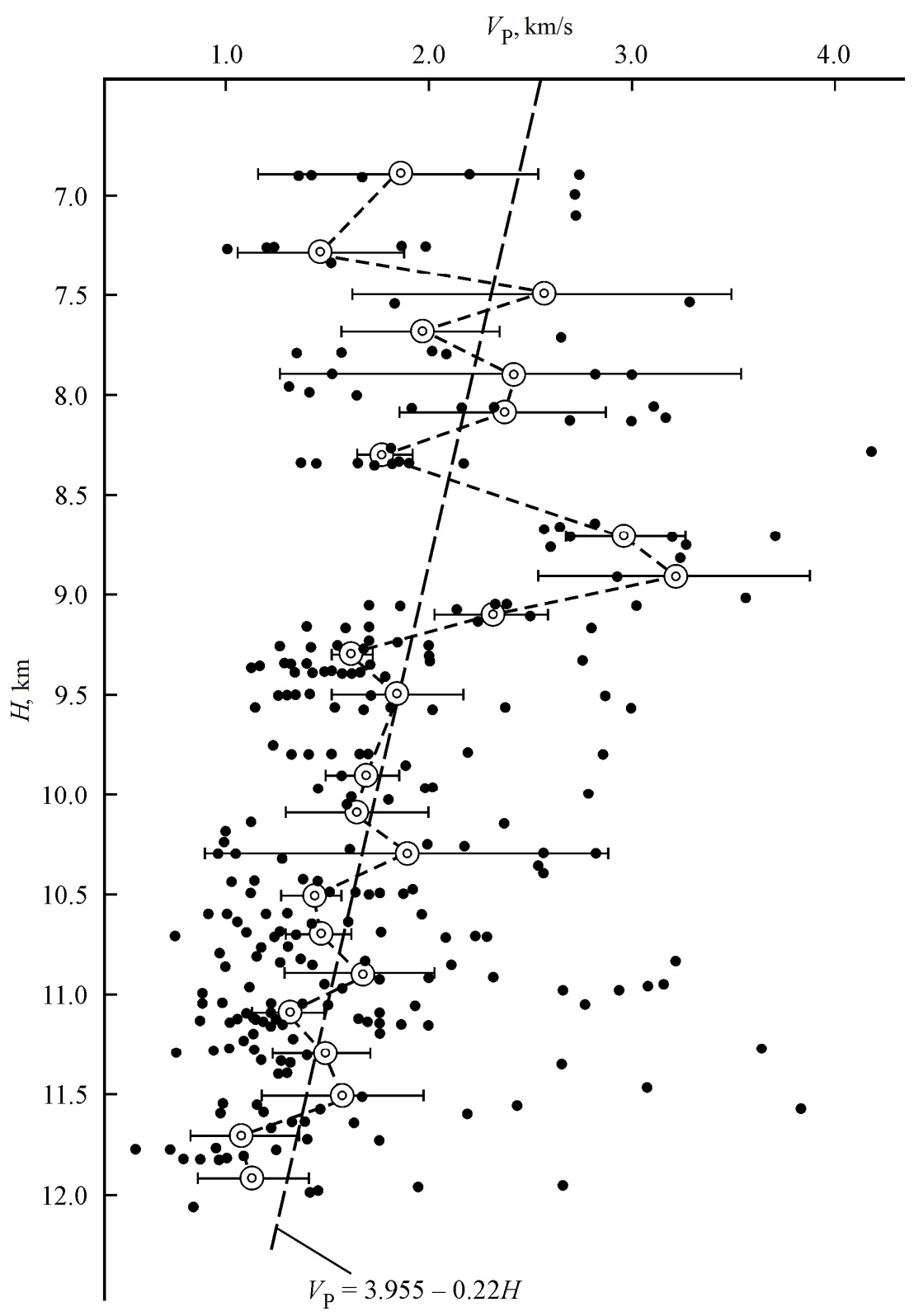

Fig. 5 Compression wave velocities VP in the samples of gneissgranite rocks from the SG-3 Archaean section (6905-12050 m) (Gorbatsevich et al., 1997).

The average velocity of compression waves calculated by mineral composition in gneisses is 6.38 $\pm 0.16 \mathrm{~km} / \mathrm{s}$, and that of shear waves - 3.52 $\pm 0.14 \mathrm{~km} / \mathrm{s}$. For schists, the average compression velocity is $6.40 \pm 0.13 \mathrm{~km} / \mathrm{s}$, the shear velocity -3.46 $\pm 0.09 \mathrm{~km} / \mathrm{s}$. The average velocities of compression and shear waves for amphibolites are $6.84 \pm 0.13 \mathrm{~km} / \mathrm{s}$ and $3.82 \pm 0.08 \mathrm{~km} / \mathrm{s}$, respectively. The velocity characteristics of rocks calculated by their mineral composition are quite close to the data received by geophysical methods. Some difference between the calculated and geophysical data can be explained by the presence of fluids in rocks in-situ. The results obtained by the calculated method can provide a basis for assessing the degree of reflection-passage of seismic waves at the interfaces of the predominant rocks in the vicinity of the SG-3 well. The relations between the compression and shear moduli in different rocks show the same trends as the average velocities. Their values are similar in schists and gneisses and higher in amphibolites. The data obtained will also help assess stress changes along the SG-3 well.

\section{ACKNOWLEDGMENTS.}

The authors are grateful to V.R. Vetrin for the useful remarks and discussion of the results obtained. This work was supported by the Russian Foundation for Basic Research (grants No. 13-05-00125-a, 16-05-00026-a). 


\section{REFERENCES}

Aleksandrov, K.S. and Ryzhova, T.V.: 1961, The elastic properties of crystals. Sov. Phys. Crystallogr., 6, 228252.

Allison, I.S. and Palmer, D.F. (Eds.): 1980, Geology: The science of a changing Earth. McGraw-Hill Book Co.

Bayanova, T.B., Pozhilenko, V.I., Smol'kin, V.F., Kudryashov, N.M., Kaulina, T.V. and Vetrin, V.R.: 2002, Catalogue of geochronological data on the northern-eastern Baltic shield. Apatity, Kola Sci. Centre of RAS, 53 pp., (in Russian).

Belikov, B.P., Aleksandrov, K.S. and Ryzhova, T.V.: 1970, Elastic properties of rock forming minerals and rocks. Moscow, Nauka, 276 pp., (in Russian).

Berckhemer, H., Rauen, A., Winter, H., Kern, H., Kontny, A., Lienert, M., Nover, G., Pohl, J., Popp, T., Schult, A., Zinke, J. and Soffel, H.C.: 1997, Petrophysical properties of the 9-km-deep crustal section at KTB. J. Geophys. Res., 102 (B8), $18337-18361$. DOI: 10.1029/96JB03396

Berry, L.G., Mason, B. and Dietrich, R.V.: 1983, Mineralogy. W.H. Freeman and Company. San Francisco.

Christensen, N.I. and Mooney, W.D.: 1995, Seismic velocity structure and composition of the continental crust: a global view. J. Geophys. Res., 100 (B7), 9761-9788. DOI: 10.1029/95JB00259

Crampin, S.: 1985, Evaluation of anisotropy by shear-wave splitting. Geophysics, 50, 1, $142-152$.

DOI: $10.1190 / 1.1441824$

Digranes, P., Kristoffersen, Y. and Karajev, N.: 1996, An analysis of shear waves observed in VSP data from the superdeep well at Kola, Russia. Geophys. J. Int., 126, $2,545-554$.

DOI: 10.1111/j.1365-246X.1996.tb05309.x

Glebovitsky, V.A.: 1973, Evolution aspects of metamorphic processes in mobile regions. Leningrad: Nauka, 127 pp., (in Russian).

Golovataya, O.S., Gorbatsevich, F.F., Kern, H. and Popp, T.: 2006, Properties of some rocks from the section of the Kola ultradeep borehole as a function of the P-T parameters. Izvestiya, Physics of the Solid Earth, 42, 11, 865-876. DOI: 10.1134/S1069351306110012

Gorbatsevich, F.F.: 1999, Acoustic polarization method for determining elastic symmetry and constants of anisotropy in solid media. Ultrasonics, 37, 4, 309-319. DOI:10.1016/S0041-624X(98)00064-X

Gorbatsevich, F.F.: 2003, Decompaction mechanism of deep crystalline rocks under stress relief. Tectonophysics, $370,1-4,121-128$.

DOI: $10.1016 / \mathrm{S} 0040-1951(03) 00181-1$

Gorbatsevich, F.F.: 2009, Acoustopolariscopy of minerals and rocks. VDM Verlag, Saabrucken.

Gorbatsevich, F.F. (Ed).: 2015, Structure, properties, state of rocks and geodynamics in the geospace of the Kola superdeep borehole (SG-3). St. Petersburg: Nauka, 366 pp., (in Russian).

Gorbatsevich, F.F., Smirnov, Yu.P. and Medvedev, R.V.: 1997, Elastic-anisotropic properties of the core from the cross-section of the Kola super deep (SD-3): Measurements by means of acoustopolariscopy. Sci. Drill, 6, 103-111.

Gorbatsevich, F.F. and Trishina, O.M.: 2017, Petrophysical properties of the Pechenga rocks of different metamorphic facies. Acta Geodyn. Geomater., 14, 4(188), 409-423. DOI: 10.13168/AGG.2017.0022
Handbook (cadastre) of physical properties of rocks: 1975. Moscow, Nedra, 279 pp., (in Russian).

Hanski, E.J., Huhma, H., Smol'kin, V.F. and Vaasjoki, M.: 1990, The age of ferropicritic volkanites and comagmatic Ni-bearing intrusions at Pechenga, Kola Peninsula. U.S.S.R. Geol. Soc. Finl. Bull, 62, 123133.

Kern, H., and Schmidt, R.: 1990, Physical properties of KTB core samples at simulated in situ conditions. Sci. Drill., 1, 217-223.

Kern, H., Mengel, K., Strauss, K.W., Ivankina, T.I., Nikitin, A.N. and Kukkonen, I.T.: 2009, Elastic wave velocities, chemistry and modal mineralogy of crustal rocks sampled by the Outokumpu scientific drill hole: Evidence from lab measurements and modeling: Phys. Earth Planet. Inter., 175, 151-166. DOI: 10.1016/j.pepi.2009.03.009

Kern, H., Popp, T., Gorbatsevich, F., Zharikov, A., Lobanov, K.V. and Smirnov, Yu.P.: 2001, Pressure and temperature dependence of $\mathrm{Vp}$ and $\mathrm{Vs}$ in rocks from the superdeep well and from surface analogues at Kola and the nature of velocity anisotropy. Tectonophysics, 338, 2, 113-134. DOI: 10.1016/S0040-1951(01)00128-7

Kovalevskiy, M.V.: 2009, The automated software and hardware complex Acoustpol: Tutorial. Apatity, "K \& M" Publ., 54 pp., (in Russian).

Kozlowsky, Ye.A. (Ed.): 1987, The super-deep well of Kola Peninsula, Springer, Berlin, Heidelberg, New York, Tokyo.

Kretz, R.: 1983, Symbols for rock-forming minerals. Amer. Mineral., 68, 277-279.

Orlov, V.P. and Laverov, N.P. (Eds.): 1998, Kola Superdeep. Scientific results and research experience, MF Technoneftegas, Moscow, 260 pp., (in Russian).

Patent No. 1281993, USSR: 1987, MKI G01N 29/04. Acoustopolariscope for measuring elasticity of solid media samples, Byull. Izobret., 1, (in Russian).

Patent No. 785737, USSR: 1980, MKI G01N 29/04. Transducer of shear ultrasonic waves. - Byull. Izobret., 45, (in Russian).

Petrov, V.P.: 1999, Metamorphism of the Early Proterozoic of the Baltic Shield, Apatity, KSC RAS Publ., 325 pp., (in Russian).

Rudnick, R.L. and Fountain, D.M.: 1995, Nature and composition of the continental crust: a lower crustal perspective, Rev. Geophys., 33, 3, 267-309. DOI: 10.1029/95RG601302

Sharov, N.V., Mitrofanov, F.P., Verba, M.L and Gillen, C. (Eds.): 2005, The lithosphere structure of the Russian part of the Barents Region. Karelian Science Centre of RAS, Petrozavodsk, 318, (in Russian).

Shurkliff, W.A.: 1962, Polarized light. Harvard University, Press.

Smithson, S.B., Wenzel, F., Ganchin, Y.V. and Morozov, I.B.: 2000, Seismic results at Kola and KTB deep scientific boreholes: velocities, reflections, fluids, and crustal composition, Tectonophysics, 329, 1-4, 301317. DOI: 10.1016/S0040-1951(00)00200-6

Trčková, J., Živor, R., Kazansky, V.I., Lobanov, K.V., Zharikov, A.V. and Smirnov, Y.P.: 2002, Comparison of elastic properties of the Kola Superdeep borehole core samples and their surface analogues obtained by static and dynamic measurements. Acta Montana, Ser. A, 21 (125), 27-54. 